\title{
A 31 Year-Old Brazilian Man with Exanthematous Lesions
}

Giliane de Souza Trindade ${ }^{1 *}$, Maria Isabel Maldonado Coelho Guedes ${ }^{2}$, Galileu Barbosa Costa ${ }^{1}$, Poliana de Oliveira Figueiredo ${ }^{1}$, Jônatas Santos Abrahão ${ }^{1}$, Erna Geessien Kroon ${ }^{1}$ and Flávio Guimarães da Fonseca ${ }^{3}$

${ }^{1}$ Laboratório de Vírus, Departamento de Microbiologia, Instituto de Ciências Biológicas, Universidade Federal de Minas Gerais, Brazil

2Laboratório de Pesquisa em Virologia Animal, Escola de Veterinária, Universidade Federal de Minas Gerais, Brazil

${ }^{3}$ Laboratório de Virologia Básica e Aplicada, Departamento de Microbiologia, Instituto de Ciências Biológicas, Universidade Federal de Minas Gerais, Brazil

*Corresponding author: Giliane de Souza Trindade, Laboratório de Vírus, Departamento de Microbiologia, Instituto de Ciências Biológicas, Universidade Federal de Minas Gerais, Brazil, Tel: +553134092747; Fax: +553134436482; E-mail: giliane@icb.ufmg.br

Received date: 15 July 2014; Accepted date: 12 August 2014; Published date: 16 August 2014

Copyright: (c) 2014 Trindade GDS, et al. This is an open-access article distributed under the terms of the Creative Commons Attribution License, which permits unrestricted use, distribution, and reproduction in any medium, provided the original author and source are credited.

\section{Description}

A 31 year-old man, previously healthy Brazilian farm worker presented to the local public health clinic with a five day history of flulike symptoms, such as headache, malaise and myalgia, and with cutaneous lesions distributed on both hands and forearms. The patient also reported high fever and axillary lymphadenopathy on his right side. Peripheral erythema was also observed on the same arm. The lesions were in multiple stages of development ranging from papules to blister-like vesicles, pustules and ulcers. Some lesions had a purulent secretion. The patient reported direct contact with dairy cows (handling and milking), that were presenting with the same kind of ulcerative lesions. The patient received analgesics for the pain and fever and also antibiotics for topical use on the pustular lesions. Although he was not hospitalized, he was unable to work for 5-6 days, when papules and vesicular lesions turned pustules and ulcers. Around 8 days later he returned to the clinic and the pustules and ulcers had started to heal developing into black scars. The patient reported he was feeling better and was able to return to work. What is your diagnosis?

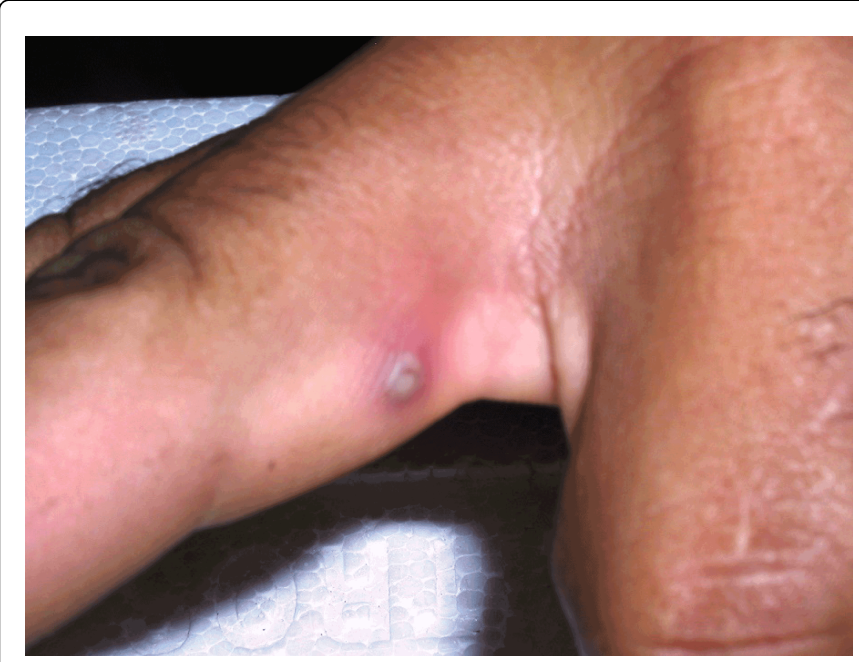

Figure 1: Vesicular lesion on hand of dairy farm worker.

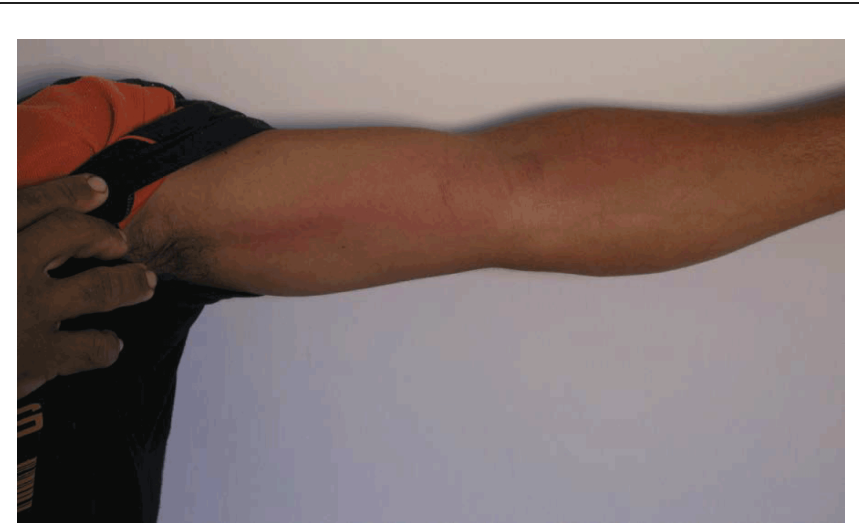

Figure 2: Axillary lymphaenopathy.

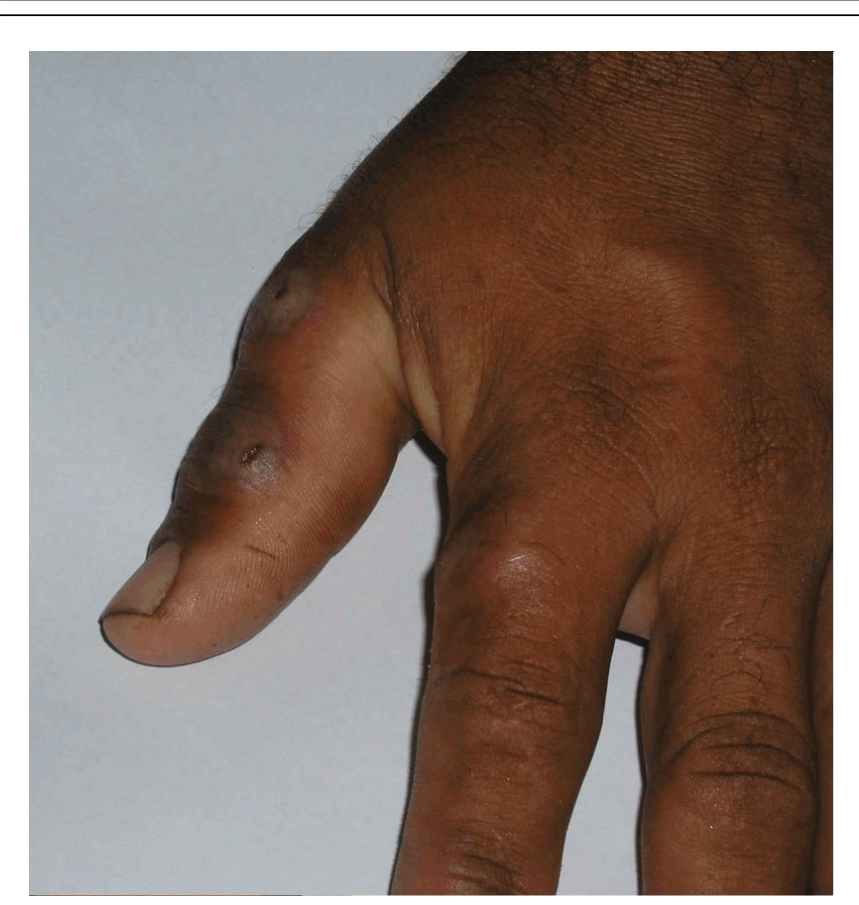

Figure 3: Pustules with umbilicated aspect on hand of dairy farm worker. 
Citation: Trindade GDS, Guedes MIMC, Costa GB, Figueiredo PDO, Abrahão JS, et al. (2014) A 31 Year-Old Brazilian Man with Exanthematous Lesions. J Vaccines Vaccin 5: i101. doi:10.4172/2157-7560.1000i101

Page 2 of 2

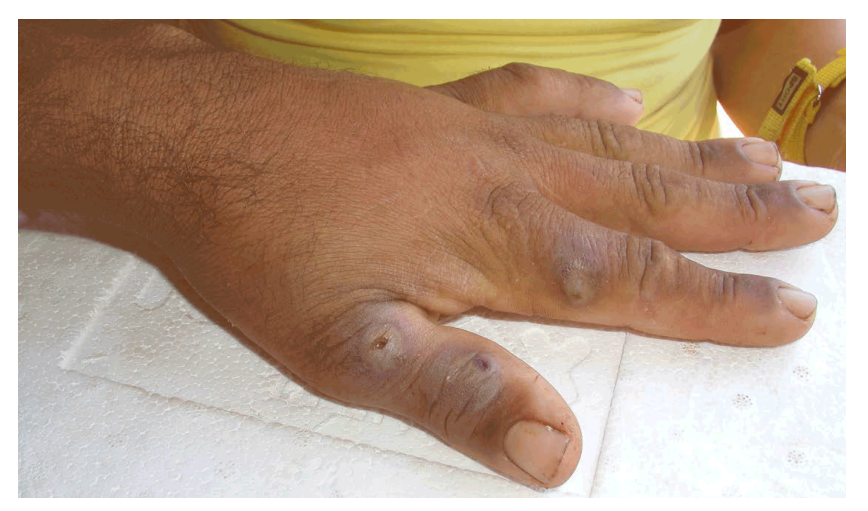

Figure 4: Pustules lesions on hand of dairy farm worker showing a central umbilicated pattern. 